Canad. Math. Bull. Vol. 43 (1), 2000 pp. 115-125

\title{
Perfect Non-Extremal Riemann Surfaces
}

\author{
Paul Schmutz Schaller
}

\begin{abstract}
An infinite family of perfect, non-extremal Riemann surfaces is constructed, the first examples of this type of surfaces. The examples are based on normal subgroups of the modular group PSL $(2, \mathbb{Z})$ of level 6. They provide non-Euclidean analogues to the existence of perfect, non-extremal positive definite quadratic forms. The analogy uses the function syst which associates to every Riemann surface $M$ the length of a systole, which is a shortest closed geodesic of $M$.
\end{abstract}

\section{Introduction}

(a) In the geometry of numbers, a well-known result of Voronoï [22] states that a positive definite quadratic form is extremal if and only if it is perfect and eutactic (see for example Gruber/Lekkerkerker [10] and Martinet [13] for more on this subject). Extremal positive definite quadratic forms correspond to extremal lattice sphere packings (see in particular Conway/Sloane [7]).

Many problems in the classical (Euclidean) geometry of numbers have their analogues in hyperbolic geometry. Let $T_{g}, g \geq 2$, be the Teichmüller space of closed Riemann surfaces of genus $g$, the surfaces being equipped with a complete hyperbolic metric (a metric of constant curvature -1). Let syst be the function on $T_{g}$ which associates to $M \in T_{g}$ the length of a systole of $M$ (a systole is a shortest closed geodesic). Then $M_{0} \in T_{g}$ is an extremal surface if syst has a local maximum in $M_{0}$. This is a non-Euclidean analogue of extremal lattice sphere packings (for closed Riemann surfaces of genus 1 the two concepts coincide). It has been introduced in Schmutz ([18], [19], see also the recent survey paper [21]). Extremal surfaces are also the subject of [15], see further Bavard [4], Quine/Zhang [16].

(b) In order that a positive definite quadratic form $f$ is extremal, it is not sufficient that $f$ is perfect. Voronoï [22] had already announced the example of a perfect non-extremal form of dimension 6. Such an example has eventually been described by Barnes [2], [3], answering a question of Coxeter [8]. Also, of the 33 different perfect forms of dimension 7, only 30 are extremal, see Conway/Sloane [6].

The aim of this paper is to provide non-Euclidean examples, namely to construct perfect non-extremal Riemann surfaces. Perfect surfaces are those which are determined by their set of systoles, see Section 2 for the precise definition.

In Section 3, I construct a family of infinitely many perfect non-extremal Riemann surfaces. The construction is based on normal subgroups of the modular group. More precisely, let $G$ be a torsion-free normal subgroup of $\operatorname{PSL}(2, \mathbb{Z})$ of finite index and of level 6 . The corresponding surface $\mathbb{H} / G(\mathbb{H}$ is the hyperbolic plane) has genus 1 and a number $n=n(G)$ of cusps. Replace the $n$ cusps in $\mathbb{H} / G$ by $n$ simple closed geodesics of the same length $12 z$ such that the automorphism group of $H / G$ is preserved; denote by $R_{G}(z)$ this

Received by the editors April 8, 1998; revised October 1, 1998.

AMS subject classification: Primary: 11H99; secondary: 11F06, $30 \mathrm{~F} 45$.

(C)Canadian Mathematical Society 2000. 
surface with boundary. Let $D_{G}(z)$ be the double of $R_{G}(z), D_{G}(z)$ is then a closed Riemann surface. $D_{G}(z)$ is contained in a 2-parameter family $A_{G}$ of closed surfaces $\left(A_{G}\right.$ corresponds to the 2-dimensional family of (2,2,2,3)-quadrilateral groups); one parameter is the length $z$, the second parameter is provided by a simultaneous twist deformation along the $n$ simple closed geodesics of length $12 z$. In $A_{G}$ one finds an, up to isometry, unique closed surface $M_{G}$ which has $10 n$ systoles of length $12 z$. We shall see that $M_{G}$ is perfect, but not extremal.

At the end of the paper I add some remarks concerning possible generalizations of this construction.

(c) The term "eutactic" (introduced by Coxeter [8]) for positive definite quadratic forms (or for lattice sphere packings) is not easy to define. However, it has been shown by Ash [1] that eutactic forms correspond to critical points of the packing function, which is a topological Morse function. I have shown in [20] (see also [21]) that also syst is a topological Morse function for Riemann surfaces. Therefore, we can say that the above described examples are perfect Riemann surfaces which are not critical points of syst.

(d) Let $\mathcal{G}$ be the set of torsion-free normal subgroups of $\operatorname{PSL}(2, \mathbb{Z})$ of finite index and of level 6. $\mathcal{G}$ can be interpreted in a Euclidean context. The elements of $\mathcal{G}$ correspond to normal subgroups of the $(2,3,6)$ triangle group (which uniformize Euclidean tori). Coxeter/Moser [9]) have classified all Euclidean tori which can be obtained by these subgroups and I shall use their classification in the present context.

An element $G \in \mathcal{G}$ will be characterized by the number $n$ of cusps of $\mathbb{H} / G$ (this is however not a $1-1$ classification). For example, $\mathcal{G}$ contains the principal congruence subgroup $\Gamma(6)$ (which has index 72 in $\operatorname{PSL}(2, \mathbb{Z})$ ) and $\mathbb{H I} / \Gamma(6)$ has 12 cusps. $\mathcal{G}$ contains exactly five elements with a smaller index in $\operatorname{PSL}(2, \mathbb{Z})$ than $\Gamma(6)$, the corresponding surfaces have $1,3,4,7$, and 9 cusps, respectively. I further note that it follows by a result of Zograf [23] and by the main result of Luo/Rudnick/Sarnak [12] that $G \in \mathcal{G}$ is not a congruence subgroup (of PSL $(2, \mathbb{Z})$ ) for $n \geq 37$ (this does not mean, of course, that all elements of $\mathcal{G}$ with $n \leq 36$ are congruence subgroups).

Acknowledgment I thank Jack Quine for helpful discussions.

\section{Definition of Perfect Non-Extremal Surfaces}

\section{Definitions}

(i) A surface $M$ is a Riemann surface of constant curvature - 1 . If $M$ is a surface with boundary, then, by definition, the boundary components are simple closed geodesics, called boundary geodesics. If $M$ is compact without boundary, then $M$ is called a closed surface.

(ii) Denote by $\mathbb{H}$ the upper halfplane. For a complete surface $M$ I also write $M=\mathbb{H} / \Gamma$ where $\Gamma$ is a Fuchsian group which uniformizes $M$.

(iii) A systole of a surface $M$ is a shortest closed geodesic of $M . S(M)$ denotes the set of systoles of $M$.

(iv) Let $\mathcal{M}$ be a closed surface. Denote by $T(\mathcal{M})$ the Teichmüller space of $\mathcal{M}$. Denote by syst the function

$$
\text { syst : } T(\mathcal{M}) \longrightarrow \mathbb{R}
$$


which associates to every $M \in T(\mathcal{M})$ the length of a systole of $M$. Let $M_{0} \in T(\mathcal{M})$. Then $M_{0}$ is called an extremal surface if syst has a local maximum in $M_{0}$.

(v) Let $\mathcal{M}$ be a closed surface. Let $M_{0} \in T(\mathcal{M})$. Then $M_{0}$ is called a perfect surface if the following condition holds.

There exists an open neighborhood $U$ of $M_{0}$ in $T(\mathcal{M})$ such that

a) the length functions of the elements of $S\left(M_{0}\right)$ parameterize $U$ and

b) $S\left(M_{0}\right) \neq S(M)$ for every $M \in U \backslash\left\{M_{0}\right\}\left(S(M)\right.$ and $S\left(M_{0}\right)$ are understood as sets of marked geodesics).

\section{Construction of the Examples}

\section{Definitions}

(i) Let $\mathcal{G}$ be the set of torsion-free normal subgroups of the modular group $\operatorname{PSL}(2, \mathbb{Z})$ of finite index and of level 6 (the level is defined in the sense of Wohlfahrt, see for example [14, p. 147]; see also the proof of Theorem 1 below for a geometric characterization). Let $G \in \mathcal{G}$. Then I write $G=G(n)$ if $H / G$ has $n$ cusps.

(ii) Let $\mathcal{N}=\left\{b^{2}+b c+c^{2}: b, c \in \mathbb{Z}, b \geq c \geq 0, b+c>0\right\}=\{1,3,4,7,9,12,13, \ldots\}$.

Remark Compare the introduction for some comments on $\mathcal{G}$.

\section{Theorem 1}

(i) Let $G=G(n) \in \mathcal{G}$. Then $\mathbb{H} / G$ has genus 1 and $n \in \mathcal{N}$.

(ii) Let $n \in \mathcal{N}$. Then there exists $G(n) \in \mathcal{G}$.

Proof Since $G(n)$ has level 6 , around each cusp of $\mathbb{H} / G$, there are exactly 12 different triangles of angles $\pi / 2, \pi / 3,0$. It follows that the area of $\mathbb{H} / G$ is $2 n \pi$ which implies that $\mathbb{H} / G$ has genus 1 . Replace the triangles of angles $\pi / 2, \pi / 3,0$ by triangles of angles $\pi / 2, \pi / 3$, $\pi / 6$. Thereby, $\mathbb{H} / G$ is transformed into an Euclidean torus. These Euclidean tori have been classified in Coxeter/Moser [9] and the theorem follows by their classification.

Remark Let $n \in \mathcal{N}$. Then $G(n)$ is not unique if the representation $n=b^{2}+b c+c^{2}$, $b \geq c \geq 0$, is not unique. $G(1)$ is however unique and $\mathbb{H} / G(1)$ is the so-called modular torus.

Lemma 2 Let $G \in \mathcal{G}$. Then $G$ is a normal subgroup of $G(1)$.

Proof Obvious since the automorphism group of $\mathbb{H} / G$ acts transitively on the cusps.

\section{Definitions}

(i) Let $z \in \mathbb{R}, z \geq 0$. Let $Q(z)$ be a hyperbolic quadrilateral with three angles $\pi / 2$ and one angle $\pi / 3$ such that one of the sides of the quadrilateral, which joins two angles $\pi / 2$, has length $z$. 

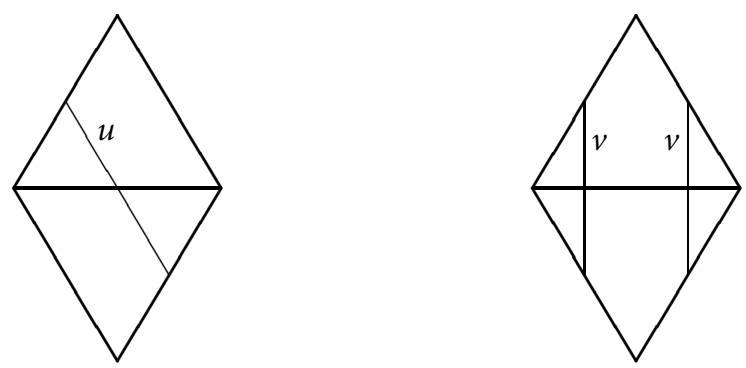

Figure 1: The modular torus with the canonical triangulation (thick lines), opposite sides have to be identified. On the left hand side, a closed geodesic $u$ with $N(u)=2$, on the right hand side a closed geodesic $v$ with $N(v)=4$.

(ii) Take six copies of $Q(z)$ such that they form a right-angled hexagon, the latter is denoted by $H(z)$.

\section{Remarks}

(i) $Q(z)$ is, up to isometry, uniquely determined for every non-negative real $z$. Note that for $z=0$ we obtain a hyperbolic triangle with angles $\pi / 2, \pi / 3,0$, which is denoted by $Q(0)$.

(ii) $H(0)$ is a triangle, its three angles being zero.

\section{Definitions}

(i) Let $G \in \mathcal{G}$. Then $\mathbb{H} / G$ is triangulated into triangles of angles $\pi / 2, \pi / 3,0$. This is called the $(2,3,6)$-triangulation. This triangulation induces a new triangulation by triangles of type $H(0)$. It will be called the canonical triangulation. (The $(2,3,6)$-triangulation is the (first) barycentric subdivision of the canonical triangulation.)

(ii) Let $u$ be a closed geodesic of $\mathbb{H} / G$. Then the canonical triangulation of $H / H / G$ separates $u$ into a number of geodesic segments, denote by $N(u)$ this number (see Figure 1 and Figure 2 for examples).

(iii) The length of a closed geodesic $u$ will be denoted by $L(u)$.

Lemma 3 Let $u$ be a closed geodesic in the modular torus $\mathbb{H} / G(1)$. If $N(u)<6$, then

$$
2 \cosh (L(u) / 2) \in\{3,6,7\} .
$$

Proof If $u$ has self-intersections, then the component of $\mathbb{H} / G(1) \backslash u$ which contains the cusp, is homeomorphic to a horodisc so that $N(u) \geq 6$. Therefore, we can assume that $u$ is simple and passes through two fixed points of the hyperelliptic involution of $H / G(1)$; these fixed points are the centres of the sides of the triangles of the canonical triangulation. It follows (compare Figure 1) that, up to isometry, there is a unique possibility for $N(u)=2$ and there are two possibilities for $N(u)=4$ (one of them is the geodesic $u$ with $N(u)=2$ passed twice). The lemma follows by an easy calculation (for formula of hyperbolic trigonometry see for example [5]). 


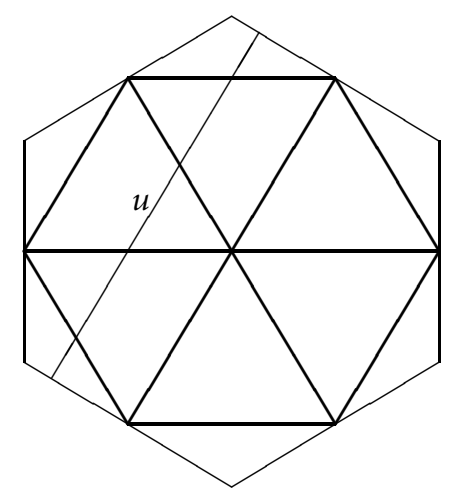

Figure 2: The surface $\mathbb{H} / G(4)$ with the canonical triangulation (thick lines), opposite sides have to be identified; $u$ is a closed geodesic with $N(u)=4$.

Corollary 4 Let $G(n) \in \mathcal{G}$. Let $u$ be a closed geodesic of $\mathbb{H} / G$. If $N(u)<6$, then $n \in$ $\{1,3,4\}$.

Proof By Lemma 2, $u$ is induced by a closed geodesic $u_{0}$ of the modular torus and $N\left(u_{0}\right) \leq$ $N(u)<6$. We therefore can apply Lemma 3.

Assume that $n>4$. Then $\mathrm{HI} / G(n)$ contains an embedded part as in Figure 2 such that the opposite sides (in Figure 2) are disjoint. It follows by Lemma 3 that $N(u)<6$ is impossible.

Definition (i) Let $G \in \mathcal{G}$. Let $z \in \mathbb{R}, z>0$. Replace each triangle $Q(0)$ of the $(2,3,6)$ triangulation of $\mathrm{H} / G$ by a quadrilateral $Q(z)$. Denote by $R_{G}(z)$ the corresponding surface with boundary.

(ii) Denote by $2 x$ the length of a boundary geodesic of $R_{G}(z)$ (note that $x=6 z$ ).

(iii) A common orthogonal of $R_{G}(z)$ is a simple geodesic which is a common orthogonal of two boundary geodesics of $R_{G}(z)$.

(iv) Denote by $\tau$ the sum of the lengths of the two sides of $Q(z)$ which enclose the angle $\pi / 3$ of $Q(z)$. Let $C$ be the sum of the lengths of the four sides of $Q(z)$. Put $t / 2=C-\tau-z$ $(t / 2$ is the length of a side of $Q(z))$.

(v) Denote by $\mathcal{T}$ the set of the common orthogonals ( of $R_{G}(z)$ ) of length $t$, corresponding to the sides of the hexagons $H(z)$ (induced by the triangles $H(0)$ of the canonical triangulation of $\mathrm{H} / G)$.

Lemma 5 Let $G(n) \in \mathcal{G}$ and let $R_{G(n)}(z), z>0$, be the corresponding surface with boundary.

(i) The shortest common orthogonals in $R_{G(n)}(z)$ are the $3 n$ elements of $\mathcal{T}$.

(ii) Among all common orthogonals of $R_{G(n)}(z)$ which are not elements of $\mathcal{T}$, let $v$ be one of shortest length. Then the length of $v$ is $2 \tau$.

(iii) Let $w$ be a common orthogonal of $R_{G(n)}(z)$ which is strictly longer than $2 \tau$. Then the length of $w$ is at least $\theta$ with $\cosh \theta=\cosh x \sinh ^{2} t-\cosh ^{2} t$. 


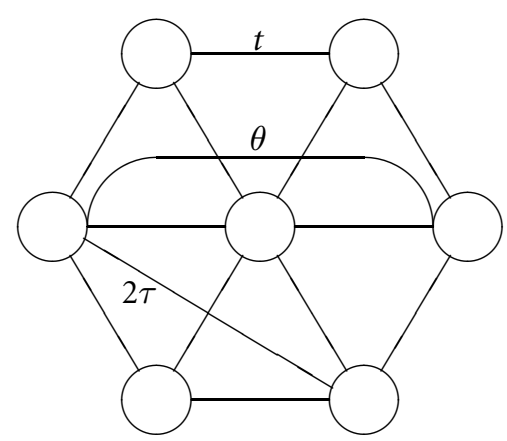

Figure 3: A part of $R_{G}(z)$ with right-angled hexagons and common orthogonals of length $t, 2 \tau$ and $\theta$, respectively. (The circles correspond to boundary geodesics of $R_{G}(z)$.)

Proof The canonical triangulation of $\mathbb{H} / G(n)$ induces a partition of $R_{G(n)}(z)$ into rightangled hexagons. The lemma follows, compare Figure 3.

Definition Let $G \in \mathcal{G}$, let $R_{G}(z)$ be the corresponding surface with boundary. Let $D_{G}(z)$ be the double of $R_{G}(z)$. Denote by $Z$ the set of simple closed geodesics of $D_{G}(z)$ which were boundary geodesics of $R_{G}(z)$. (The double $D_{G}(z)$ of $R_{G}(z)$ is defined such that $D_{G}(z)$ has an orientation reversing involution fixing pointwise the elements of $Z$.)

Remark By construction, $D_{G}(z)$ is a closed surface and the automorphism group of $D_{G}(z)$ contains a subgroup $\Sigma$ of a $(2,2,2,3)$-quadrilateral group. Let $T_{G}$ be the Teichmüller space of $D_{G}(z)$. Then $T_{G}$ contains a family, of two real parameters, of surfaces such that their automorphism group contains $\Sigma$; one parameter of this family is $z$, the other parameter is provided by a simultaneous twist deformation of the same amount (and in the same direction) along all elements of $Z$.

Definition (i) Denote by $A_{G}$ the family of two real parameters in $T_{G}$ which is described in the previous remark. Denote by $\Sigma$ the subgroup of a $(2,2,2,3)$-quadrilateral group which characterizes the family $A(G)$.

(ii) I now define a particular element $M_{G}$ in $A_{G}$. Let $q \in \mathcal{T}$; $q$ joins two boundary geodesics of $R_{G}(z)$ which are denoted by $z_{1}$ and $z_{2}$. Denote by $Y(q)$ the unique embedded pair of pants (a surface of genus zero with three boundary geodesics) of $R_{G}(z)$ which contains $z_{1}, z_{2}$ and $q$. Let $X(q)$ be the double of $Y(q)$, embedded in $D_{G}(z) ; X(q)$ has genus 1 and two boundary geodesics. Then $M_{G}$ is defined by the property that $X(q)$ has five geodesics of length $2 x$ which are systoles of $X(q)$.

Define $X(\mathcal{T})=\{X(q): q \in \mathcal{T}\}$.

Remark Up to isometry, $M_{G}$ is uniquely defined in $A_{G}$, compare [18]. Note however that the (common) sign of the twist deformations along the elements of $Z$ is not well-defined. 
Lemma 6 Let $G \in \mathcal{G}$ and let $M_{G}$ be the corresponding closed surface. Then $M_{G}$ has three sets of simple closed geodesics of length $2 x$ which are invariant with respect to $\Sigma$, one of these sets is $Z$. The two other sets have $3 n$ and $6 n$ elements, respectively.

Proof $\Sigma$ acts transitively on the elements of $X(\mathcal{T})$ (since $\Sigma$ acts transitively on the elements of $\mathcal{T})$; by Lemma $5, X(\mathcal{T})$ has $3 n$ elements. Let $X(q) \in X(\mathcal{T})$. Then $X(q)$ has five simple closed geodesics of length $2 x$. One of them intersects all other four, this induces a set $V$ of $3 n$ simple closed geodesics of length $2 x$, invariant with respect to $\Sigma$. $X(q)$ contains further two elements of $Z$. The last two closed geodesics of length $2 x$ of every $X(q) \in X(\mathcal{T})$ are contained in a set $W$ of $6 n$ elements, invariant with respect to $\Sigma$.

Definition Define the sets $V$ and $W$ of simple closed geodesics in $M_{G}$ of length $2 x$ as in the proof of Lemma 6.

Lemma 7 Let $X(q) \in X(\mathcal{T})$. Let $v \in V, w \in W, z \in Z$ be three simple closed geodesics of length $2 x$ in $X(q)$. Let $\gamma$ be defined by

$$
\cos \gamma=\frac{\cosh x}{1+\cosh x}
$$

Then both $w$ and $z$ intersect $v$ in the angle $\gamma$.

Proof This follows by a calculation (compare [18]).

Lemma 8 Let $G \in \mathcal{G}$ and let $M_{G}$ be the corresponding closed surface. Let $\theta$ be defined as in Lemma 5. Then

(i) $x \sim 3.17575$

(ii) $t \sim 1.6206$

(iii) $2 \tau \sim 3.5809$

(iv) $\theta \sim 4.8499$.

Proof The lengths $t, \tau$, and $\theta$ depend on $x$. The length $x$ can be calculated using the identities

$$
\cosh t=\frac{\cosh (x / 3)}{\cosh (x / 3)-1}
$$

provided by the hexagons $H(z)$, and

$$
\sinh (t / 2)=\sinh (x / 2) \sin \gamma
$$

provided by an analysis of an element of $X(\mathcal{T})$; the latter identity, together with Lemma 7 , implies

$$
\cosh t=\frac{\cosh x(1+3 \cosh x)}{(1+\cosh x)^{2}}
$$

and $x$ is determined by (1) and (2). 
Theorem 9 Let $G(n) \in \mathcal{G}, n>7$, and let $M=M_{G(n)}$ be the corresponding closed surface. Then $M$ has exactly $10 n$ systoles of length $2 x$.

Proof Let $u$ be a systole of $M$. Assume that $u$ does not intersect an element of $Z$. Then $u \in Z$ or $u$ has the same length as a systole of $R_{G(n)}(z)$. Since $n>7$, a systole of $R_{G(n)}(z)$ intersects at least six right-angled hexagons $H(z)$ (by Corollary 4) so that it is longer than $2 x$. Therefore, $u \in Z$.

Assume now that $u$ intersects $2 m>0$ elements of $Z$. Then $L(u) \geq 2 m t$ by Lemma 5 which implies by Lemma 8 that $m=1$ (since $2 t>x$ ). Therefore, $u$ is separated by $Z$ into two parts $u_{1}$ and $u_{2}$, each one is homotopic to a common orthogonal in a copy of $R_{G(n)}(z)$. By Lemma 5 and Lemma 8, one of them, $u_{1}$ say, is homotopic to an element of $\mathcal{T}$ (since $2 \tau>x$ ), and $u_{2}$ is then homotopic to a common orthogonal of length $t$ or of length $2 \tau$ (since $t+\theta>2 x$ ). The latter case is only possible for $n \leq 7$ (which is excluded by hypothesis). Therefore, $u_{1}$ and $u_{2}$ are both homotopic to an element of $\mathcal{T}$. Since $n>7$ it follows (compare the proof of Corollary 4) that in $M$, there exists a subsurface $X(q)$ containing $u$. This implies $L(u)=2 x$. We have therefore proved that the systoles of $M$ have length $2 x$. The number $10 n$ follows by Lemma 6 .

Theorem 10 Let $G(n) \in \mathcal{G}, n>7$, and let $M=M_{G(n)}$ be the corresponding closed surface. Then $M$ is a perfect closed surface which is not extremal.

Proof By Theorem 9, the systoles of $M$ are identified. Let $z \in Z$. Then there are exactly six elements of $X(\mathcal{T})$ which contain $z$. The six elements of $V$ in these six elements of $X(\mathcal{T})$ are the boundary geodesics of an embedded subsurface $S$ of $M$ of genus 1 . In the interior, $S$ has 13 systoles of $M$, namely $z$, six elements of $V$ and six elements of $W$. The corresponding length functions determine $S$, compare [17], [18]. It follows that $M$ is perfect.

To prove that $M$ is not extremal, requires some calculation. Let $\xi$ be a vector in the tangent space of $M$ which is induced by a twist deformation of the same amount (and in the same direction) along all elements of $V$. Denote by $\xi(u)$ the real number obtained by applying $\xi$ to the length function of a simple closed geodesic $u$. Then, see for example [11],

$$
\xi(u)=\sum_{i} \cos \gamma_{i}
$$

where the sum is over all directed angles $\gamma_{i}$ in the intersection points of $u$ with the elements of $V$. Since the elements of $V$ are mutually disjoint, we have

$$
\xi(v)=0, \quad \forall v \in V
$$

By Lemma 7 it follows that

$$
\xi(z)=6 \cos \gamma>0, \quad \forall z \in Z
$$

(we measure the angles clockwise (in Figure 4) from the elements of $V$ to $z$ ). Let $w \in W$. Then $w$ is intersected by exactly six elements of $V$. It follows by Figure 4 (and by the symmetry of $M$ ) that the six angles of the corresponding intersections are (recall that we measure the angles clockwise) $2 \gamma,-\gamma_{1},-\gamma_{2},-\gamma,-\gamma_{2},-\gamma_{1}$. 


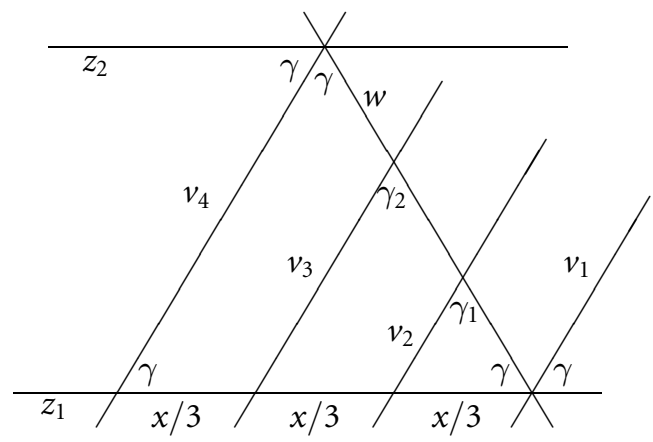

Figure 4: An element $w \in W$ intersects $z_{i} \in Z, i=1,2$, and six elements of $V$, four of them ( $v_{k}$, $k=1, \ldots, 4)$ are drawn.

Again by Figure 4, we have, for $j=1,2$,

$$
\cos \gamma_{j}=\sin ^{2} \gamma \cosh (j x / 3)-\cos ^{2} \gamma
$$

Since we know $x$ and $\gamma$ (by Lemma 8 and Lemma 7), we can calculate $\cos \gamma_{j}, j=1,2$. A calculation then gives

$$
\xi(w)=-\cos \gamma-2 \cos \gamma_{2}-2 \cos \gamma_{1}+\cos (2 \gamma) \sim 1.46, \quad \forall w \in W
$$

We have therefore shown that $\xi(u)>0, \forall u \in Z \cup W$.

Let $\zeta$ be the vector in the tangent space of $M$ which is induced by a twist deformation of the same amount (and in the same direction) along all elements of $Z$ such that

$$
\zeta(v)=2 \cos \gamma>0, \quad \forall v \in V
$$

(this is possible by Lemma 7). It follows that the span of $\xi$ and $\zeta$ (in the tangent space of $M)$ contains a vector $\eta$ such that

$$
\eta(u)>0, \quad \forall u \in Z \cup V \cup W .
$$

This implies that $M$ is not a local maximum for syst.

Corollary 11 Let $m$ be any positive integer. Then there exists an integer $g \geq 2$ such that in the Teichmüller space $T_{g}$ of closed surfaces of genus $g$, there exist more than m mutually non-isometric surfaces which all are perfect, but not extremal. Moreover, these surfaces can be 
chosen such that the length of their systoles is the same and the number of their systoles is the same.

Proof Take $G(n) \in \mathcal{G}$ such that $n$ has more than $m$ different representations $n=b^{2}+b c+c^{2}$, $b \geq c \geq 0, b, c$ integers. Each different representation gives a different surface $M_{G(n)}$. By Theorem 10 they are all perfect, but not extremal.

Remark I add some remarks concerning possible generalizations of the construction of perfect non-extremal surfaces given in this paper.

(i) Here is another idea for a possible proof of Corollary 11. Let $G(n) \in \mathcal{G}$. In the definition of $M=M_{G(n)}$ we have seen that the (common) direction of the twist deformations along the elements of $Z$ is not well-defined, they can be all positive or all negative, say (but the amount of the twist deformations is well-defined). Choose a subset $Z^{\prime} \subset Z$. Construct a new surface $M\left(Z^{\prime}\right)$ by inverting the twist deformation along the elements of $Z^{\prime}$. Then $M\left(Z^{\prime}\right)$ has still $10 n$ closed geodesics of length $2 x$ and they are still the systoles since the proof of Theorem 9 goes through. Also, $M\left(Z^{\prime}\right)$ is still perfect by the argument in the proof of Theorem 10. But $M\left(Z^{\prime}\right)$ may be extremal.

(ii) Let $\mathcal{G}(N)$ be the set of the torsion-free normal subgroups of the modular group of finite index and of level $N$ (for a fixed integer $N \geq 7$ ). As in the case $N=6$, we can also construct a closed surface $M_{G}$ for every $G \in \mathcal{G}(N)$. It is very probable that the proofs of Theorem 9 and Theorem 10 also work in this case if we exclude those $G \in \mathcal{G}(N)$ which produce only a few cusps; for example for big $N$, we have to exclude the principal congruence subgroup $\Gamma(N)$ (remember that in the case $N=6$ we had to exclude $n \leq 7$ ).

(iii) I finally note that we could also work with a torsion-free subgroup $\Gamma$ of the modular group of finite index which is not normal, but shares the property of normal subgroups that around each cusp in $H / \Gamma$, there is the same number of triangles with angles $\pi / 2, \pi / 3,0$.

\section{References}

[1] A. Ash, On eutactic forms. Canad. J. Math. 29(1977), 1040-1054.

[2] E. S. Barnes, The perfect and extreme senary forms. Canad. J. Math. 9(1957), 235-242.

[3] The complete enumeration of extreme senary forms. Philos. Trans. Roy. Soc. London Ser. A 249(1957), 461-506.

[4] C. Bavard, Systole et invariant d'Hermite. J. Reine Angew. Math. 482(1997), 93-120.

[5] P. Buser, Geometry and spectra of compact Riemann surfaces. Birkhäuser, 1992.

[6] J. H. Conway and N. J. A. Sloane, Low-dimensional lattices, III. Perfect forms. Proc. Roy. Soc. London Ser. A 118(1988), 43-80.

[7] $\longrightarrow$ Sphere packings, lattices and groups. Second ed., Springer, 1993.

[8] H. S. M. Coxeter, Extreme forms. Canad. J. Math. 3(1951), 391-441.

[9] H. S. M. Coxeter and W. O. J. Moser, Generators and relations for discrete groups. Fourth ed., Springer, 1980.

[10] P. M. Gruber and C. G. Lekkerkerker, The geometry of numbers. Second ed., North-Holland, 1987.

[11] S. Kerckhoff, The Nielsen realization problem. Ann. of Math. 117(1983), 235-265.

[12] W. Luo, Z. Rudnick and P. Sarnak, On Selberg's eigenvalue conjecture. Geom. Funct. Anal. 5(1995), $387-401$.

[13] J. Martinet, Les réseaux parfaits des espaces euclidiens. Masson, Paris, 1996.

[14] M. Newman, Integral matrices. Academic Press, 1972.

[15] J. R. Quine and P. Sarnak (eds.), Extremal Riemann surfaces. Contemp. Math. 201, Amer. Math. Soc., 1997.

[16] J. R. Quine and P. L. Zhang, Extremal symplectic lattices. Israel J. Math. (to appear).

[17] P. Schmutz, Die Parametrisierung des Teichmüllerraumes durch geodätische Längenfunktionen. Comment. Math. Helv. 68(1993), 278-288.

[18] Riemann surfaces with shortest geodesic of maximal length. Geom. Funct. Anal. 3(1993), 564-631.

[19] Systoles on Riemann surfaces. Manuscripta Math. 85(1994), 429-447. 
[20] P. Schmutz Schaller, Systole is a topological Morse function for Riemann surfaces. Preprint, 1997.

[21] Geometry of Riemann surfaces based on closed geodesics. Bull. Amer. Math. Soc. 35(1998), 193-214.

[22] G. Voronoï, Sur quelques propriétés des formes quadratiques positives parfaites. J. Reine Angew. Math. 133(1908), 97-178.

[23] P. G. Zograf, Small eigenvalues of automorphic Laplacians in spaces of parabolic forms. J. Soviet Math. 36(1987), 106-114.

Université de Neuchâtel

Institut de mathématiques

Rue Emile-Argand 11

CH-2007 Neuchâtel

Switzerland

Paul.Schmutz@maths.unine.ch 